\title{
Characterising doctor-parent communication in counselling for impending preterm delivery
}

\author{
J A F Zupancic, H Kirpalani, J Barrett, S Stewart, A Gafni, D Streiner, M L Beecroft, \\ P Smith
}

Arch Dis Child Fetal Neonatal Ed 2002;87:F1 13-F1 17

See end of article for authors' affiliations

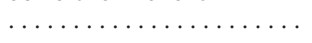

Correspondence to: Dr Kirpalani, Department of Pediatrics, McMaster Health Sciences Centre, McMaster University, 1200

Main Street West,

Hamilton, Ontario,

Canada L8S 4J9;

kirpalan@fhs.csu.

mcmaster.ca

Accepted

7 February 2002

\begin{abstract}
Objective: To examine the counselling of women admitted to hospital in preterm labour. Such women and their partners are often asked to participate in difficult decisions including mode of delivery, fetal monitoring, and resuscitation.

Study design: Questionnaire based descriptive study.

Study setting: A tertiary level perinatal referral centre.

Patients: Forty nine women in preterm labour at 22-30 weeks gestation, admitted in two separate periods between March 1997 and May 1999.

Intervention and outcome measure: Within 24 hours of counselling, parents were asked to complete a questionnaire assessing recall of the management plan, desire for involvement in decision making, anxiety, and feelings of control over their health. A parallel questionnaire was completed by the clinicians.

Results: Parents and clinicians on recall agreed well about obstetric issues but poorly about neonatal issues. Overall $27 \%$ of parents felt: "I would prefer to have the doctors advise me, rather than asking me to decide". In $79 \%$ of cases, clinicians believed parents preferred advice rather than to make decisions, but in $45 \%$ of these, they misidentified those who wished to make their decisions. Anxiety levels for one third of the mothers were high, and associated with poorer concordance of recall between parents and clinicians.

Conclusions: Serious deficiencies exist in parent-clinician encounters during extremely preterm labour. Concordance between parents and clinicians is poor and anxiety very high. A quarter of parents appear to prefer to relinquish decision making autonomy, but clinicians cannot correctly identify this subgroup. Standardised counselling in the perinatal period, using formal decision aids, should be investigated.
\end{abstract}

S ociety has placed increasing emphasis on patient involvement in clinical decision making in most Western medical settings. ${ }^{1-3}$ Despite this emphasis, the process of disclosing information for the purposes of assisting parents in giving informed consent to perinatal treatment has been poorly studied.

Parents faced with imminent delivery of a preterm infant are often given complex information, in the expectation that they will make their preferences known in order to help make difficult decisions on mode of delivery, intrapartum monitoring, and neonatal resuscitation. Previous investigations suggest that extraneous factors may compromise the parents' ability to rationally assess competing risks versus benefits. These factors include inconsistency of neonatal outcome data provided to parents by clinicians, ${ }^{4-11}$ potentially impaired ability to retain and to understand this information in stressful periods such as labour, ${ }^{12}$ time constraint, anxiety, ${ }^{13-15}$ and even the willingness or confidence of parents to be involved in decision making. ${ }^{16}$

To understand further the cumulative effects and interactions between these factors, we carried out a questionnaire based descriptive study of parents facing imminent preterm delivery. The objectives were to determine (a) the concordance between parental and clinician perceptions of information given, $(b)$ parental preferences for involvement in perinatal decision making, and $(c)$ the impact on the process of anxiety, sociodemographic characteristics, and beliefs about control over personal health.

\section{METHODS}

Study setting and participants

The study took place in the high risk obstetrics service at McMaster Health Sciences Centre in Hamilton, Ontario. This
40 bed unit serves as the tertiary level referral unit for a regional population of 1.8 million people. There are 3500 admissions annually, with about 200 births occurring at 23-30 weeks gestation. The study took place in two separate recruitment periods, from July 1997 to March 1998 and from November 1998 to May 1999, to coincide with availability of study personnel.

Participants were 49 pregnant women, and their partners, who had been admitted to the high risk perinatal service with a diagnosis of preterm labour at 23-30 weeks gestation. In addition, experienced neonatal fellows or attending neonatal physicians who had discussed potential problems of preterm delivery with the parents were surveyed.

\section{Instruments}

Two questionnaires, for parents and physicians, were developed specifically for the study through literature review and expert consensus by a panel consisting of an obstetrician, two neonatologists, two nurses (obstetric and neonatal), a psychologist, a biostatistician, and an epidemiologist with experience in decision analysis. All non-recall components of the parental questionnaire had been previously validated on parents of infants in the neonatal intensive care unit and found to have good face validity, comprehensibility, and reliability. ${ }^{16}$ The factual recall components were pretested for readability and comprehensibility on ten high risk obstetric

Abbreviations: STAI, state-trait anxiety inventory; MHLC, multidimensional health locus of control 
patients who were not part of the main sample. Face validity was good, and any misunderstood or ambiguous items were rewritten.

The parental questionnaire consisted of 72 items in four sections. In the first section, parents were asked to report their recall of the information given to them by their doctors after admission. Specific items included gestational age, pregnancy complications, neonatal problems, probability of survival, and probability of completely normal childhood development. In each case, parents first chose from a menu of gestational ages, then provided their recall of physician predicted survival and morbidity estimates in $10 \%$ probability increments, or lay descriptions of various medical conditions. In addition, parents were asked to recall whether a management plan/decision for the intrapartum period was made, who determined that plan/decision, and which elements of intrapartum intervention (such as caesarean section, monitoring, and neonatal resuscitation) would be undertaken. Finally, this section asked parents to report, on a six point Likert scale, their perceptions of coercion, complexity of the information, and the desire to be involved or to delegate decision making to the doctor.

In a second section, parents were asked to complete the 20 item state component of the Spielberger state-trait anxiety inventory (STAI). ${ }^{17}$ This tool measures current levels of anxiety, as opposed to a tendency towards anxiety at baseline. The third section of the parental questionnaire consisted of the multidimensional health locus of control (MHLC). ${ }^{18}$ The MHLC assesses the degree to which a person believes that control of their health is in their own hands, in the hands of powerful others such as doctors, or determined by chance. Both the Spielberger STAI and the MHLC have been widely used in the psychology literature and found to have excellent reliability and validity. ${ }^{17} 18$

The fourth and final section of the questionnaire included questions on sociodemographics and previous experience with prematurity or pregnancy complications. In total, the instrument took about 15 minutes to complete. The full questionnaire is available from the authors.

A second, 16 item questionnaire was developed for the neonatal physician who had discussed the pregnancy complications with the parents after admission to hospital. The questions parallelled those for the first part of the parental questionnaire, by asking the doctor to recall which pregnancy complications, neonatal conditions, estimates of survival, morbidity, and gestation were discussed, as well as the details of the peripartum management plan/decision. In addition, doctors were asked their perceptions of the parents' comprehension and preferences for involvement in decision making.

\section{Manoeuvre}

After admission to the high risk obstetric service with a diagnosis of preterm labour, it is standard practice at the study centre for women and their families to be counselled on the nature of the pregnancy complications, maternal and infant prognosis, and management options in the perinatal period. This information is provided during initial consultation with the admitting obstetrician or house staff, and subsequently by a neonatologist or neonatal fellow in a separate consultation. The format of the consultation was left to the discretion of the clinician. In the 24 hour period after these meetings, parents and the neonatal clinicians involved were asked by the study nurse to complete the questionnaire and to return it in a sealed envelope. If more than one parent was present at the meeting, the parents were asked to complete the questionnaire together, with separate information being collected only for sociodemographic characteristics. Assistance with any reading difficulties was offered, but no parents availed themselves of this opportunity.

Families were asked to participate in the study after the discussion with the clinician. A one page letter accompanying
Table 1 Sociodemographic and pregnancy characteristics of participating families

\begin{tabular}{ll}
\hline Married (\%) & 61 \\
first pregnancy (\%) & 37 \\
Other children at home (\%) & 51 \\
Hospitalised with previous pregnancy (\%) & 22 \\
Previous premature infant (\%) & 10 \\
Mother & \\
Median age (years) & 28 \\
Proportion < 19 years (\%) & 6 \\
Employed (\%) & 67 \\
High school education (\%) & 82 \\
Father & \\
Median age (years) & 30 \\
Employed (\%) & 74 \\
High school education (\%) & 69 \\
Income < $\$ 20,000$ & 25 \\
Gestational age at admission (\%) & \\
$22-26$ weeks & 33 \\
$27-30$ weeks & 63 \\
Preterm labour & 39 \\
Preterm rupture of membranes (\%) & 31 \\
Pregnancy induced hypertension (\%) & 6 \\
Placental abruption (\%) & 6 \\
\hline & \\
\hline
\end{tabular}

the questionnaire detailed the purpose of the study. The option to decline participation or to leave some questions unanswered was emphasised. The study was approved by the Institutional Research Group at McMaster University.

\section{Data analysis}

Concordance between parents and clinicians for individual variables was analysed with Cohen's weighted $\kappa$ statistic. Global agreement was assessed by an agreement score, which was the sum of all exact agreements between parents and clinicians. Determinants of agreement were assessed by performing linear regression of the agreement score on sociodemographic characteristics, STAI, and MHLC scales.

\section{RESULTS}

\section{Study participants}

A sample of convenience consisting of 49 families was obtained from those women admitted with a diagnosis of preterm labour. Women were not recruited for several reasons, of which the most common were unavailability of study personnel at the time of the consultation, progression to delivery before consultation, or advanced labour. Only six patients who were approached refused to participate, and one failed to complete the questionnaire. Table 1 gives the characteristics of the participating families and their pregnancies. Of these consultations, $37 \%$ were completed by attending doctors and the remainder by fellows.

\section{Maternal psychological characteristics}

The median score on the STAI was 44 , which is at the 77 th centile for women. ${ }^{17}$ More importantly, $30 \%$ of the mothers in the study scored above the 90th centile.

Median scores on the MHLC scales were 18 for the chance locus of control scale, 26 for the internal locus of control scale, and 19 for the powerful others locus of control scale. None of these differed from population median, ${ }^{18}$ suggesting that the participants had similar beliefs in the forces that controlled their health to the norm.

\section{Agreement between parents and clinicians}

Concordance between parents and clinicians was $76 \%$ for the decile of survival probability, but only $44 \%$ for the probability of normal development. 
Table 2 Recall by parents and doctors of information discussed pertaining to pregnancy complications

\begin{tabular}{|c|c|c|c|c|c|c|c|}
\hline & Recall by parents: & Yes & Yes & No & No & & \\
\hline Item recalled as discussed & Recall by doctor: & Yes & No & Yes & No & Concordance (\%) & p Value* \\
\hline Premature labour & & 33 & 22 & 17 & 28 & 61 & 0.180 \\
\hline Prolonged rupture of membranes & & 39 & 8 & 3 & 50 & 89 & 0.000 \\
\hline Oligohydramnios & & 3 & 19 & 6 & 72 & 75 & 0.629 \\
\hline Chorioamnionitis & & 3 & 14 & 0 & 83 & 86 & 0.023 \\
\hline Hypertension & & 8 & 3 & 0 & 89 & 97 & 0.000 \\
\hline Intrauterine growth restriction & & 6 & 14 & 3 & 78 & 84 & 0.031 \\
\hline Fetal distress & & 0 & 17 & 0 & 83 & 83 & n.c. \\
\hline Placental abruption & & 3 & 8 & 6 & 83 & 86 & 0.000 \\
\hline Placenta previa & & 11 & 3 & 0 & 86 & 97 & 0.201 \\
\hline
\end{tabular}

Table 3 Recall of parents and doctors of information discussed pertaining to neonatal problems

\begin{tabular}{|c|c|c|c|c|c|c|c|}
\hline & Recall by parents: & Yes & Yes & No & No & & \\
\hline Item recalled as discussed & Recall by doctor: & Yes & No & Yes & No & Concordance (\%) & p Value* \\
\hline Respiratory distress/apnoea & & 87 & 5 & 8 & 0 & 87 & 0.671 \\
\hline Infection & & 41 & 14 & 22 & 24 & 65 & 0.081 \\
\hline Intraventricular haemorrhage & & 53 & 18 & 13 & 16 & 69 & 0.092 \\
\hline Feeding/necrotising enterocolitis & & 39 & 34 & 13 & 13 & 53 & 0.846 \\
\hline Growth & & 11 & 26 & 26 & 37 & 48 & 0.420 \\
\hline Retinopathy of prematurity & & 37 & 11 & 13 & 39 & 76 & 0.001 \\
\hline Patent ductus arteriosus & & 16 & 16 & 18 & 50 & 66 & 0.163 \\
\hline Jaundice & & 11 & 8 & 26 & 55 & 66 & 0.218 \\
\hline Difficulty for parents to feed or hold & & 8 & 8 & 34 & 50 & 58 & 0.670 \\
\hline Was a plan made for management? & & 59 & 4 & 37 & 0 & 59 & 0.615 \\
\hline Caesarean section if necessary for baby? & & 48 & 16 & 9 & 27 & 75 & 0.006 \\
\hline Resuscitate $v$ resuscitate based on appearance? & & 71 & 11 & 7 & 11 & 82 & 0.306 \\
\hline
\end{tabular}

Tables 2 and 3 show the concordance between parents and clinicians for recall of the discussion of pregnancy complications and neonatal problems respectively. As shown, agreement for obstetric variables was good, ranging from $61 \%$ to $97 \%$, with significant $\kappa$ statistics (indicating agreement beyond chance) for all categories except prematurelabour, oligohydramnios, and placenta previa. In contrast, concordance on potential neonatal problems was generally poor, $48-87 \%$, with significant $\kappa$ statistics only for retinopathy of prematurity. Parents and clinicians agreed that a management plan had been formulated for the intrapartum period in only $59 \%$ of cases, and for decisions to undertake neonatal resuscitation in $82 \%$ of cases. However, perception that caesarean section would be undertaken for fetal indications was concordant in $75 \%$ of cases, with a significant agreement beyond chance.

In linear regression, the agreement score, consisting of all matching responses between clinicians and parents, correlated negatively with the maternal STAI score $(r=0.36$, $p=0.025$ ). Thus, mothers who had higher levels of anxiety were less likely to agree with clinician recall of the information dispensing meeting. Similarly, families with a previous premature infant were less likely to be in agreement with physician responses $(r=0.390, \mathrm{p}=0.014)$. There was no effect of maternal health locus of control or sociodemographic characteristics on concordance.

\section{Preferences for involvement}

Some $27 \%$ of the participants agreed or strongly agreed with the statement, "I would prefer to have the doctors advise me what I should do with my pregnancy, rather than asking me to decide." Physicians identified 79\% of the parents as "probably" or "definitely" preferring to be advised, rather than making a decision after being informed of the options. However, $45 \%$ of these assessments actually occurred in patients who disagreed or strongly disagreed with the statement-that is, those who wanted to make the decision primarily on their own.

\section{DISCUSSION}

In this descriptive study of parents, concordance was poor between physicians and parents about medical information discussed after admission for preterm labour. The effect was most pronounced for information on potential neonatal problems, but also extended to obstetric complications and recall of a perinatal management plan.

Deficiencies in doctor-patient communication are not unique to the discussion of preterm labour. In a seminal study, patients making decisions about adjuvant cancer treatment agreed poorly with their physicians about recurrence risks and side effects of treatment. ${ }^{19}$ In one of the few studies of informed consent in a peripartum population, when women who had consented to epidural analgesia in labour were interviewed within 48 hours of delivery, 33\% could not recall discussion of any information on risks. There was, however, a higher rate of recall of specific risks in women who had attended antenatal classes, leading the authors to suggest that information presented in labour is less well retained..$^{12}$

The cause of this poor agreement and recall is likely to be multifactorial and requires both a larger population sample and further work, but this study provides some clues. In our population, admittedly a relatively homogeneous one, there was no apparent association between sociodemographic factors and concordance. This is consistent with a previous study in a similar population, which showed no impact of sociodemographic characteristics on consent to involvement in neonatal research. ${ }^{1620}$ The health locus of control instrument that was used is well characterised, and differentiates between those who believe that they can control their own 
health and those who believe it is controlled more by external circumstances and healthcare givers. Our results showing insignificant differences from population norms suggest that the determining factors on preferences for decision making may be process related, rather than predetermined by any personal traits of the participants.

In contrast, higher maternal acute anxiety levels as measured by a standardised instrument were associated with lower levels of agreement about issues discussed. Moreover, a subset of mothers had particularly high acute anxiety levels compared with population norms. Other studies suggest that anxiety levels are raised in populations who have recently consented to medical treatment for their children, ${ }^{13}$ and higher anxiety may affect whether patients consent to treatments. ${ }^{15} 21$

In addition to concerns about recall of information and the effect of anxiety on the process, the study also suggests that the current standard of information giving is unable to accommodate patients with different preferences for involvement, and may even be described as haphazard. One quarter of participants expressed a preference to have the physician advise them on what to do with the pregnancy, rather than the physician giving them clinical information and then asking them to decide. This is consistent with a study of parents approached for consent for involvement in neonatal research. ${ }^{16}$ Although physicians as a group were aware that some parents preferred "guidance" rather than autonomy in decision making, they were only able to identify $27 \%$ of those who wanted to make the decision independently. Pronounced national differences in both the degree and types of counselling given in neonatal units across Europe ${ }^{22}$ have been attributed to cultural styles. These cultural factors also appear to affect the willingness of physicians to consider parental opinion on provision or continuation of intensive care..$^{23}$ These factors would introduce even further variance.

Several limitations of this study warrant attention. Firstly, because audiotaping of the parent-physician interviews was not feasible given the resources available for the study, we cannot comment specifically on recall or accuracy of information, but only on consistency of responses between physicians and parents. Where such studies have been performed they showed an appreciable difference between parental and physician recall of information from the taped evidence. $^{1924}$ Similarly, we cannot assess the quality of physician communication objectively without direct observation. The time lag between completion of the consultation and completion of the questionnaire may also have adversely affected recall by both parents and physicians. This delay was again unavoidable given clinician time constraints and the length of the questionnaire.

Secondly, the study was not designed to assess parents' comprehension of the facts discussed, as we felt this would impose more stress and time on the parents. However, an adequate agreement between physicians and parents as to what has been discussed during the consultation is a necessary prerequisite to a valid consent process.

Thirdly, the small sample size and relatively advantaged sociodemographic population in the study centre may limit the power of the study to detect associations between concordance and patient characteristics. A further limitation is the large number of physicians involved in the decision making process. However, this may paradoxically increase generalisability, as many physicians of varying sensitivities and skills are commonly involved in the decision making process.

Finally, we did not record certain maternal characteristics, such as the degree to which she was contracting and the associated discomfort, any drugs she may have received in labour, or underlying medical or psychiatric disorders. The underlying heterogeneity in these factors, however, more closely reflects clinical reality, in which information is often given in a hurried manner, to patients with a variety of medical conditions, or during an acute episode when delivery may be imminent.

These limitations are unlikely to affect the central findings of deficiencies in parental-physician concordance about medical aspects of pregnancy, the impact of anxiety on agreement, and the inability of physicians to correctly identify patients with alternative decision making preferences. Moreover, other investigators have shown that there is significant variability and inaccuracy among physicians with regard to morbidity and mortality outcomes of premature infants, and that these knowledge deficiencies change parental decision making. ${ }^{4-610}{ }^{11}$ Taken together, the results suggest that the current method of information giving for the purposes of informed consent for management of high risk pregnancies is flawed.

Can an alternative process for informed consent and information sharing improve accuracy, consistency, recall, and anxiety? A comparison of the theoretical models of therapeutic decision making is instructive. ${ }^{25}$ The traditional "paternalistic" model of doctor-patient communication involves authoritative decision making by the physician with little patient input, other than agreement with the doctor's recommendation. Although our results suggest that there is a subgroup of patients who may prefer such an approach, physicians were not able to identify them consistently. In contrast, in an "informed" model, the physician contributes only information, and the patient is solely responsible for deliberation and decision making. In both of these approaches, the asymmetry in roles assumed in decision making may allow misunderstandings, miscomprehension, and inadvertent intimidation to occur. A possible solution is a "shared" model, in which doctor and patient participate simultaneously and symmetrically in all facets of the decision making process. ${ }^{26}$ The literature on doctor-patient communication hints that such shared decision making is not the predominant mode of interaction in the clinical setting, with only $10 \%$ of the average interaction time across 61 studies being devoted to "partnership building", in which the doctor elicits patient involvement or facilitates through synthesis and interpretation of the discussion. ${ }^{27} 28$

In response to such deficiencies in the process, researchers and clinicians in other areas of medicine have collaborated to develop formal decision aids to facilitate shared patient and clinician decision making. The format of these instruments has ranged from simple algorithmic flip charts or decision boards ${ }^{29}$ to audiotapes, ${ }^{30}$, computer programs, and interactive videotapes. $^{31}$ Within the perinatal field, a simple, noninteractive table of outcomes stratified by gestational age has also been developed. ${ }^{32-34}$ Although studies of the effects of these have been methodologically limited, ${ }^{35}$ they do hold promise to standardise the information presented as well as to enable partnership building in the decision process. Priority should be given to further development and rigorous evaluation of such instruments for perinatology.

\section{ACKNOWLEDGEMENTS}

We gratefully acknowledge the cooperation of the perinatal and neonatal staff of McMaster Health Sciences Centre, and the families who agreed to participate at a stressful time in their lives. J A F Z was supported in part by a Clinician-Scientist Award from the Medical Research Council of Canada.

\section{Authors' affiliations}

J A F Zupancic, Department of Neonatology, Beth Israel Deaconess Medical Center, Harvard Medical School, Boston, Massachusetts, USA H Kirpalani, Department of Pediatrics, McMaster University, Hamilton, Ontario, Canada

J Barrett, Department of Nursing, McMaster University

S Stewart, Centre for Community Health and Health Evaluation

Research, British Columbia's Children's Hospital, Vancouver, British

Columbia, Canada 
A Gafni, Department of Clinical Epidemiology and Biostatistics, McMaster University

D Streiner, Department of Psychiatry, University of Toronto, Toronto Ontario, Canada

M L Beecroft, P Smith, Department of Obstetrics and Gynecology, McMaster University

\section{REFERENCES}

1 Cassileth BR, Zupkis RV, Sutton-Smith K, et al. Information and participation preferences among cancer patients. Ann Intern Med 1980;92:832-6

2 Hughes TE, Larson LN. Patient involvement in health care. A procedural justice viewpoint. Med Care 1991;29:297-303.

3 Harrison $\mathbf{H}$. The principles for family-centered neonatal care. Pediatrics 1993;92:643-50.

4 Haywood JL, Goldenberg RL, Bronstein J, et al. Comparison of perceived and actual rates of survival and freedom from handicap in premature infants. Am J Obstet Gynecol 1994;171:432-9.

5 Haywood JL, Morse SB, Goldenberg RL, et al. Estimation of outcome and restriction of interventions in neonates. Pediatrics 1998;102:e20.

6 Goldenberg RL, Nelson KG, Dyer RL, et al. The variability of viability: the effect of physicians' perceptions of viability on the survival of very low birth weight infants. Am J Obstet Gynecol 1982;143:678-84.

7 Lee SK, Penner PL, Cox M. Comparison of the attitudes of health care professionals and parents toward active treatment of very low birth weight infants. Pediatrics 1991;88:110-14

8 Haywood J, Burnham D, Goldenberg R, et al. American pediatricians underestimate survival and freedom from handicap in premature infants [abstract]. Pediatr Res 1994;34:1619A.

9 Wilson AL, Wellman LR, Fenton L, et al. What physicians know about the prognosis of preterm newborns. American Journal of Diseases in Children 1983;137:551-4.

10 Sanders MR, Donohue PK, Oberdorf MA, et al. Perceptions of the limit of viability: neonatologists' attitudes toward extremely preterm infants. J Perinatol 1995; 15:494-502.

11 de Garis C, Kuhse H, Singer P, et al. Attitudes of Australian neonatal paediatricians to the treatment of extremely preterm infants. Aust Paediatr J 1987:23:223-6.

12 Swan HD, Borshoff DC. Informed consent: recall of risk information following epidural analgesia in labour. Anaesth Intensive Care 1994;22:139-41.

13 Roling GT, Pressgrove LW, Keeffe EB, et al. An appraisal of patients' reactions to "informed consent" for peroral endoscopy. Gastrointest Endosc 1977:24:69-70.

14 Ruccione K, Kramer RF, Moore IK, et al. Informed consent for treatment of childhood cancer: factors affecting parents' decision making. J Pediat Oncol Nurs 1991;8:112-21.

15 Antrobus JH. Anxiety and informed consent. Does anxiety influence consent for inclusion in a study of anxiolytic premedication? Anaesthesia 1988;43:267-9

16 Zupancic JAF, Gillie P, Streiner DL, et al. Determinants of parental authorization for involvement of newborn infants in clinical trials. Pediatrics 1997;99:e6.
17 Spielberger C, Gorsuch R, Lushene R. STAl manual for the state-trait anxiety inventory. Palo Alto, CA: Consulting Psychologists Press Inc, 1970.

18 Wallston K, Wallston B. Development of the multidimensional health locus of control (MHLC) scales. Health Education Monographs 1978;6:161-70

19 Siminoff LA, Fetting JH, Abeloft MD. Doctor-patient communication about breast cancer adjuvant chemotherapy. J Clin Oncol 1989:7: 1 192-200.

20 Harth SC, Thong YH. Sociodemographic and motivational characteristics of parents who volunteer their children for clinical research: a controlled study. BM 1990;300:1372-5.

21 Harth SC, Johnstone RR, Thong YH. The psychological profile of parents who volunteer their children for clinical research: a controlled study. $J$ Med Ethics 1992; 18:86-93.

22 Cuttini $M$, Rebagliato $M$, Bortoli $P$, et al. Parental visiting, communication, and participation in ethical decisions: a comparison of neonatal unit policies in Europe. Arch Dis Child Fetal Neonatal Ed 1999;81:F84-91.

23 De Leeuw R. Treatment choices for extremely preterm infants: An international perspective. J Pediatr 2000;137:608-15

24 Perlman NB, Freedman JL, Abramovitch R, et al. Informational needs of parents of sick neonates. Pediatrics 1991;88:512-18.

25 Charles C, Whelan T, Gafni A. What do we mean by partnership in making decisions about treatment? BM 1999;319:780-2.

26 Charles C, Gafni A, Whelan T. Shared decision-making in the medical encounter: what does it mean? (or it takes at least two to tango). Soc Sci Med 1997;44:681-92

27 Ong LML, de Haes JCJM, Hoos AM, et al. Doctor-patien communication: a review of the literature. Soc Sci Med 1995;40:903-18.

28 Roter DL, Hall JA, Katz NR. Patient-physician communication: a descriptive summary of the literature. Patient Education and Counselling 1988;12:99-119

29 Levine MN, Gafni A, Markham B, et al. A bedside decision instrument to elicit a patient's preference concerning adjuvant chemotherapy for breast cancer. Ann Intern Med 1992;1 17:53-8.

30 O'Connor AM, Tugwell P, Wells GA, et al. Randomized trial of a portable, self-administered decision aid for postmenopausal women considering long-term preventive hormone therapy. Med Decis Making 1998; 18:295-303.

31 Gramlich EP, Waitzfelder BE. Interactive video assists in clinical decision making. Methods Inf Med 1998;37:201-5.

32 Koh TH, Casey A, Harrison H. Use of an outcome by gestation table for extremely premature babies: a cross-sectional survey of the views of parents, neonatal nurses and perinatologists. J Perinatol 2000;20:504-508.

33 Koh TH, Harrison H, Morley C. Gestation versus outcome table for parents of extremely premature infants. J Perinatol 1999;19:452-3.

34 Koh TH, Harrison H, Casey A. Prediction of survival for preterm births. Survival table was not easy to understand. BM 2000;320:647.

35 Molenaar S, Sprangers MA, Postma-Schuit FC, et al. Feasibility and effects of decision aids. Med Decis Making 2000;20:1 12-27. 